\title{
Monitoring of the Damage Evolution in Reinforced Concrete Girder by Means of Nonlinear Elastic Wave Spectroscopy
}

\author{
M. Kořenská*, M. Manychová, L. Pazdera \\ Faculty of Civil Engineering, Brno University of Technology, Czech Republic \\ *Corresponding author: korenska.m@fce.vutbr.cz \\ K. Pospíšil \\ CDV - Transport Research Centre, Brno, Czech Republic
}

DOI: $10.2478 / \mathrm{v} 10158-012-0022-3$

\begin{abstract}
Nonlinear elastic wave spectroscopy (NEWS) is a package of advanced methods of ultrasonic spectroscopy which make it possible to capture with a high level of sensitivity the formation and development of structure damage even in materially, as well as geometrically, highly complicated specimens. Concrete and reinforced concrete are classical examples of materials to which the application of conventional ultrasonic methods is very complicated. This is why they make an ideal medium for the application of non-linear ultrasonic methods. The object of this experimental study consists of the application of nonlinear ultrasonic testing to assess the structural integrity of a reinforced concrete girder which was extracted from a bridge structure during the reconstruction of the bridge. The girder was tested in three stages: prior to loading, in the course of loading, and when the loading had been completed, with the aim of identifying the parameters correlating with the girder structure integrity damage. Two nonlinear ultrasonic spectroscopy methods were applied, namely, employing one and two harmonic signals. Frequency spectra of the transmission responses were analyzed. Defects occurring in the structure under investigation give rise to heavy nonlinear effects accompanying the propagation of elastic waves, which, in the singlesignal excitement case took effect in emphasizing the odd-numbered harmonic components among the newly generated frequencies. Therefore, the amplitudes of the latter were evaluated. In the other case, two ultrasonic signals of close frequencies were applied and their difference components were evaluated. Structure integrity damage was identified in the girder by means of the frequency spectrum analysis.
\end{abstract}

KEY WORDS: Nonlinear ultrasonic spectroscopy, harmonic analysis, reinforced concrete testing.

\section{INTRODUCTION}

Most of today's non-destructive ultrasonic testing methods are based on the application of linear ultrasound excitation and on the assumption of a linear elastic continuum. These conventional ultrasonic testing methods provide an efficient and reliable tool for a variety of applications. However, there are applications where these methods encounter heavy limitations and become impracticable, for example in the cases 
of complicated material composition or defects which are undetectable by current ultrasound wavelength signals. This is why nonlinear ultrasonic testing methods started to be developed in the last two decades, spreading into the areas which are inaccessible for classical methods (Shuia et al., 2008; Aleshin \& Van Den Abeele, 2007). The principle of these methods consists of anharmonic oscillations of atoms at the defect or crack faces. The reason is that the oscillating atom energy versus displacement plot does not exactly follow the quadratic law (Claytor et al., 2009). Consequently, odd-numbered harmonics of the fundamental frequency are prevailingly generated. This phenomenon provides us with an efficient tool to discriminate between damaged and undamaged specimens (Haupert et al., 2011).

The ultrasonic defectoscopy is therefore a powerful tool to study defects and cracks in solids. In some cases, provided the method has been calibrated, the extent and type of the damage can be determined. As most of the nonlinear effects are amplitude-dependent, taking effect only after sufficiently high-amplitude exciting signals are applied, it is evident that the exciting signal amplitude plays a crucial role in the nonlinear ultrasonic testing methods. The threshold exciting amplitude, which is capable of bringing about a recordable nonlinear response, depends, above all, on the type of the material under test, the damage nature and the excitement type.

Thanks to its composition, concrete is a heavily hysteretic material, which shows a certain degree of nonlinearity even under no-load conditions (Nazarov et al., 2003; Bentahar et al., 2006; Antonaci et al., 2010). If there are defect regions in the concrete specimen under test, these regions will make additional sources of nonlinearity which exceed, by several orders of magnitude, the specimen's own nonlinearity. Under ultrasonic tests, the nonlinear behaviour of the material takes effect in a deformation of the ultrasonic waves propagating through the specimen, thus resulting in nonlinear effects in the frequency spectra of the specimen under investigation. The nonlinear effects consist of an amplitude-dependent occurrence of higher harmonic components $\left(f_{2}, f_{3}, \ldots\right)$ when a single-frequency excitation with a frequency $f_{l}$ is used, and a marked growth of odd-numbered harmonics and the formation of side bands $\left(f_{1} \pm f_{2}, f_{1} \pm 2 f_{2}, \ldots\right)$ when simultaneous excitation by two frequencies $f_{1}$ and $f_{2}$ is used (Hajek et al., 2003). Another option of time-dependent excitation is the application of an impulse signal. In this case, the application of a mechanical impulse signal may result in a substantially higher output response. Besides the generation of new harmonic components, the defect induced nonlinearity also results in a change of the specimen transfer characteristics and both of these phenomena can be analysed at a time.

Thanks to its robustness and relative simplicity, the nonlinear ultrasonic spectroscopy, NEWS, as a method based on evaluating the higher harmonic component amplitude ratios, has proved to be suitable for concrete and reinforced concrete testing. As all of the phenomena employed in the NEWS methods are amplitude dependent, we focused our attention on amplitude characteristics of higher harmonics $\left(f_{2}, f_{3}\right)$ and their ratio, $f_{n} / f_{1}$. When applying the impulse excitation, we focused on the frequency analysis of the response versus the exciting impulse intensity function.

\section{TESTED OBJECT AND EXPERIMENTAL ARRANGEMENT}

The object of our experiment was a reinforced concrete girder which had been extracted from a bridge structure in the course of its reconstruction. The girder was of KA type, its dimensions being $0.5 \mathrm{~m} \times 0.6 \mathrm{~m} \times 11.5 \mathrm{~m}$. It was brought to CDV laboratories at Tisnov to be placed on an elastic cushion, which in turn was seated on two concrete guard rails. 
A special frame had been assembled for the purpose of loading. For safety reasons, it was designed so as to allow the loading process to take place beneath the girder itself. The girder was measured in three stages: prior to loading, in the course of loading and when the loading had been completed. The objective of these measurements consisted of monitoring the structure integrity damage. The r.h.s. part of the specimen was measured at 4 points, henceforth denoted as measurement positions.
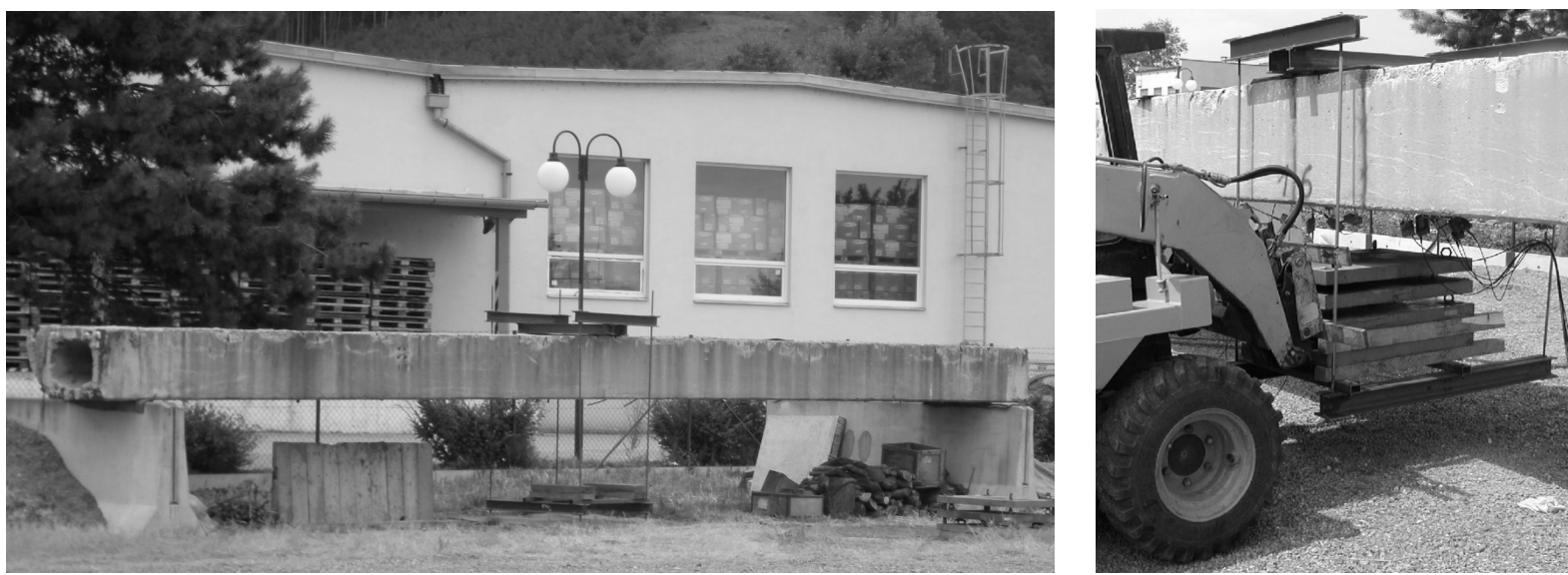

Figure 1: The girder with the loading frame (left), dynamic girder loading (right).

\section{MEASUREMENT RESULTS}

Measurement results obtained from two measurement positions differing from each other in the steel armature pattern are presented here. First, we used the single exciting signal method which is described in detail, together with the measuring apparatus, in (Korenska \& Manychova, 2010; Manychova, 2009; Korenska et al., 2008). A harmonic exciting signal of a frequency of $f=29 \mathrm{kHz}$ was applied, transmission responses of the girder, as picked up by the sensor, were analyzed (Zumpano \& Meo, 2008). The orientation of the exciter E and the sensor $\mathrm{S}$ is diagrammatically shown in Fig. 2.
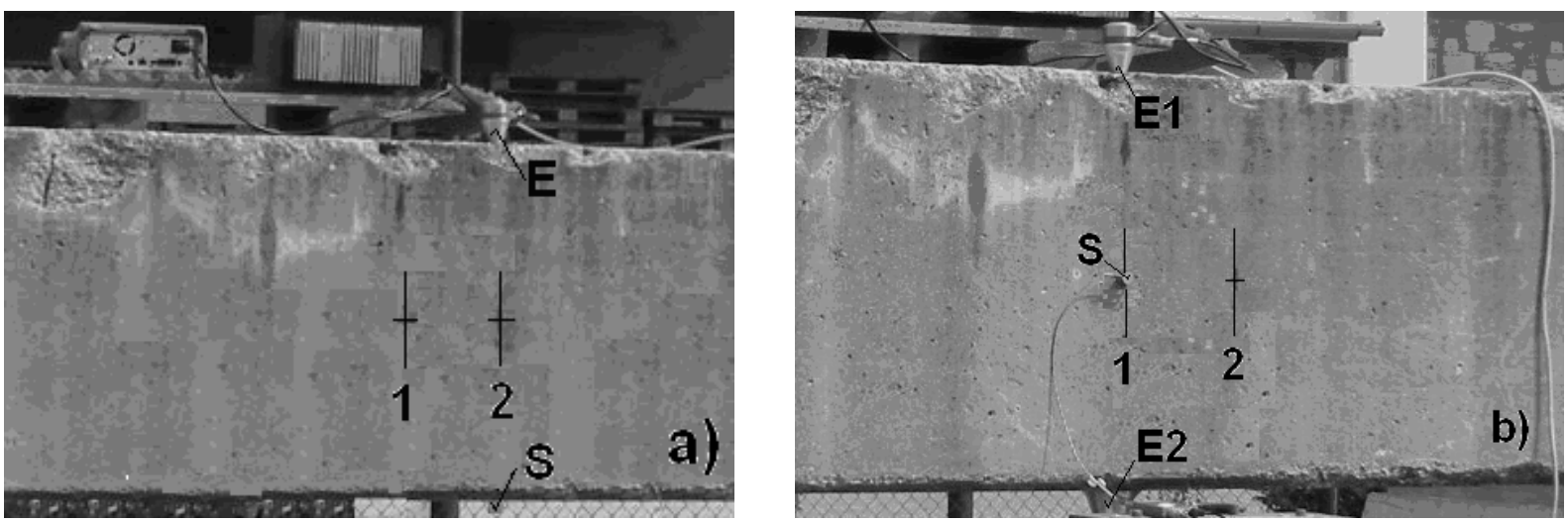

Figure 2: Schematic illustration of the measurement positions:

a) measurement on position No. 2 (single exciting signal method),

b) measurement on position No. 1 (double exciting signal method). 
During the measurement on position No. 1 (Fig. 2a) the exciter E and sensor S were placed above and under the measurement position, similarly to the situation with the measurement on position No. 2. During the measurement on position No. 2 (Fig. 2b) the exciters E1, E2 and sensor $\mathrm{S}$ were placed similarly to the situation with the measurement on position No. 1.

The measurements resulted in the transmission response frequency spectra. By way of illustration, Fig. 3 shows this frequency spectrum for No. 2 measurement position. The diagram illustrates an abrupt fall of amplitudes of even-numbered harmonic frequencies ( $f_{2}$ and $f_{4}$ ), the amplitude of the fifth harmonic $\left(f_{5}\right)$ exceeding that of the amplitude of the fourth harmonic $\left(f_{4}\right)$, providing us with information on the occurrence of structuredefect-induced nonlinear effects. This is in accordance with cited foreign sources and our knowledge from results of extensive laboratory measurements.

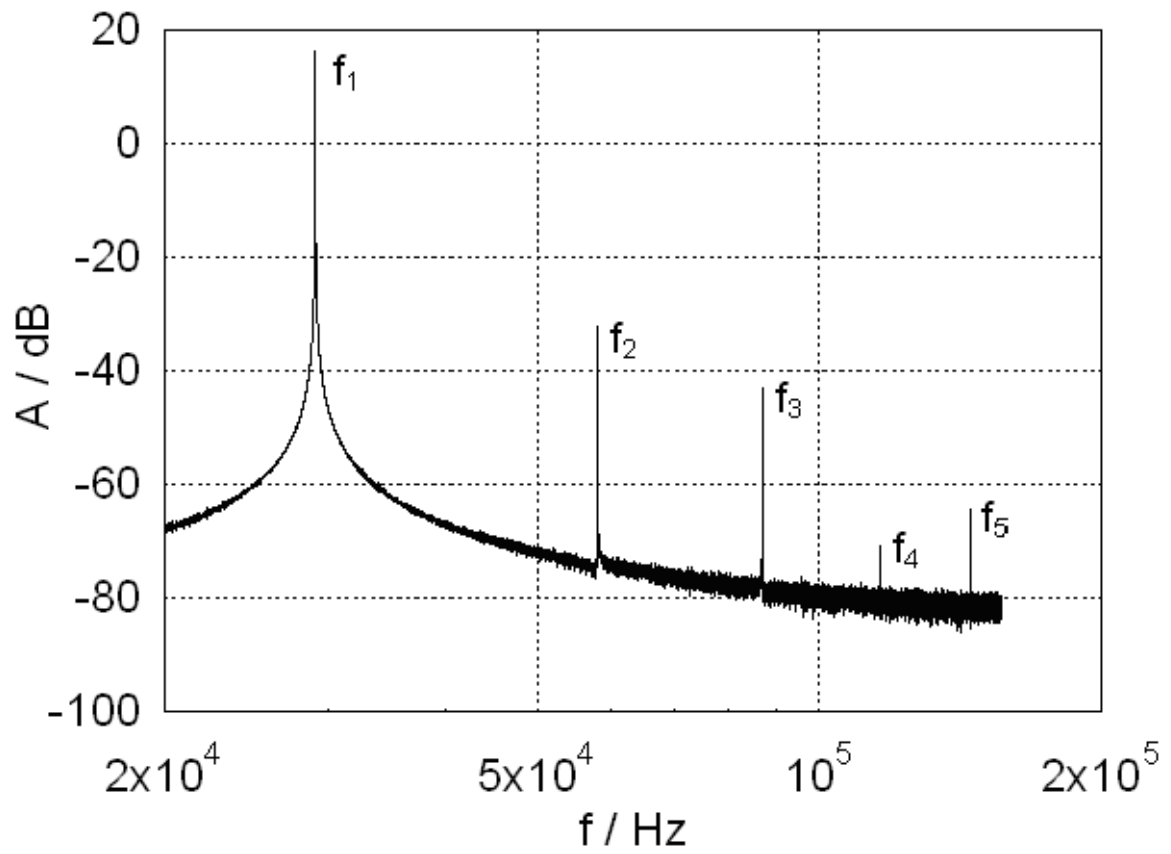

Figure 3: Frequency spectrum for No. 2 measurement position and excitation signal frequencies $29 \mathrm{kHz}$.

Fig. 4 shows the values of higher harmonic frequencies db-amplitudes relative to the $1^{\text {st }}$ harmonic (exciting frequency $f_{l}$ ) db-amplitude for No. 1 and 2 measuring positions. From the comparison of the curves we may infer that better structure quality corresponds to No. 1 measuring position. In this case, the relative amplitudes are decreasing uniformly with the harmonic frequency order number, with no nonlinear effects. 


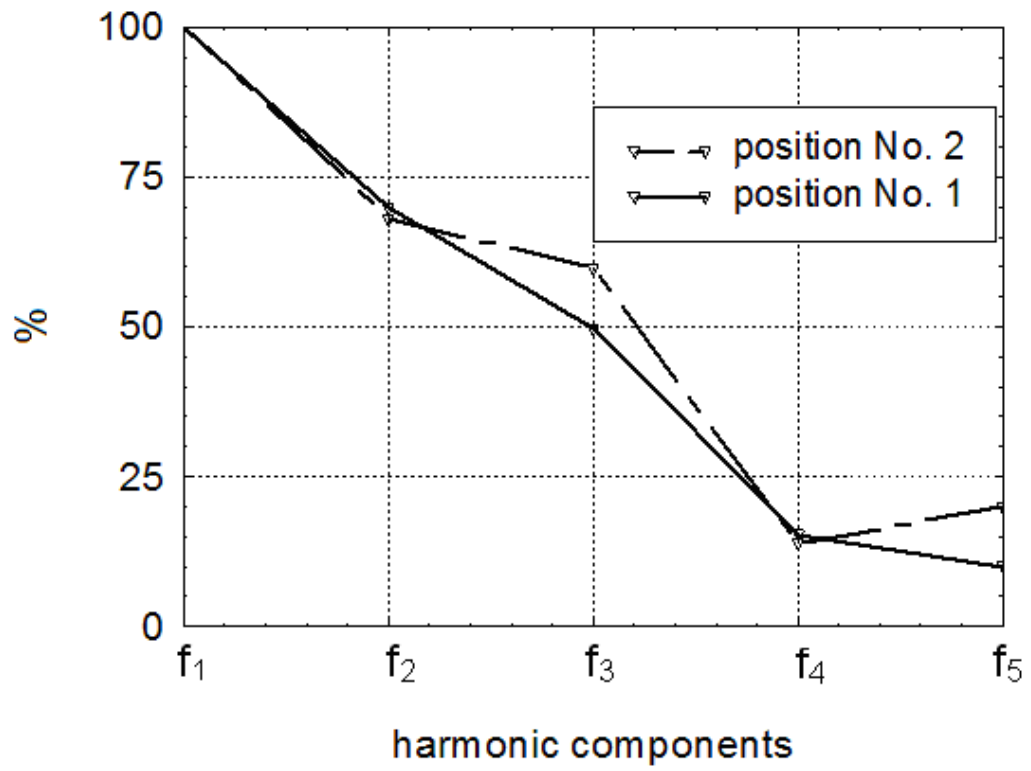

Figure 4: Higher harmonic frequency amplitudes expressed as a percentage of the first harmonic amplitude.

Furthermore, the correlation coefficient square, $r^{2}$, was evaluated. This quantity serves to evaluate the linearity of the harmonic frequency amplitude decrease curve. The correlation coefficient square nears unity if the amplitude vs. frequency plot decrease shows no nonlinear effects, which is the case of an intact structure. The more the amplitude decrease plot shape differs from the straight line, the more $r^{2}$ tends to zero. The correlation coefficient squared shows a decrease in value $\left(r^{2}=0.9216\right)$ for the second position compared to the first position $\left(r^{2}=0.9728\right)$. This parameter also exhibits higher-quality structure in the case of position No. 1. Secondly, two harmonic ultrasonic signals whose frequencies neared each other $\left(f_{1}=29 \mathrm{kHz}\right.$ and $\left.f_{2}=25 \mathrm{kHz}\right)$, were applied, see Fig. $2 \mathrm{~b}$ ). In the frequency spectrum, which corresponds to No. 2 position, Fig. 5, there occurs, beside some parasitic components, the excitation frequency difference component, $\Delta f=4 \mathrm{kHz}$. This difference component did not occur in the case of the measurement position No. 1.

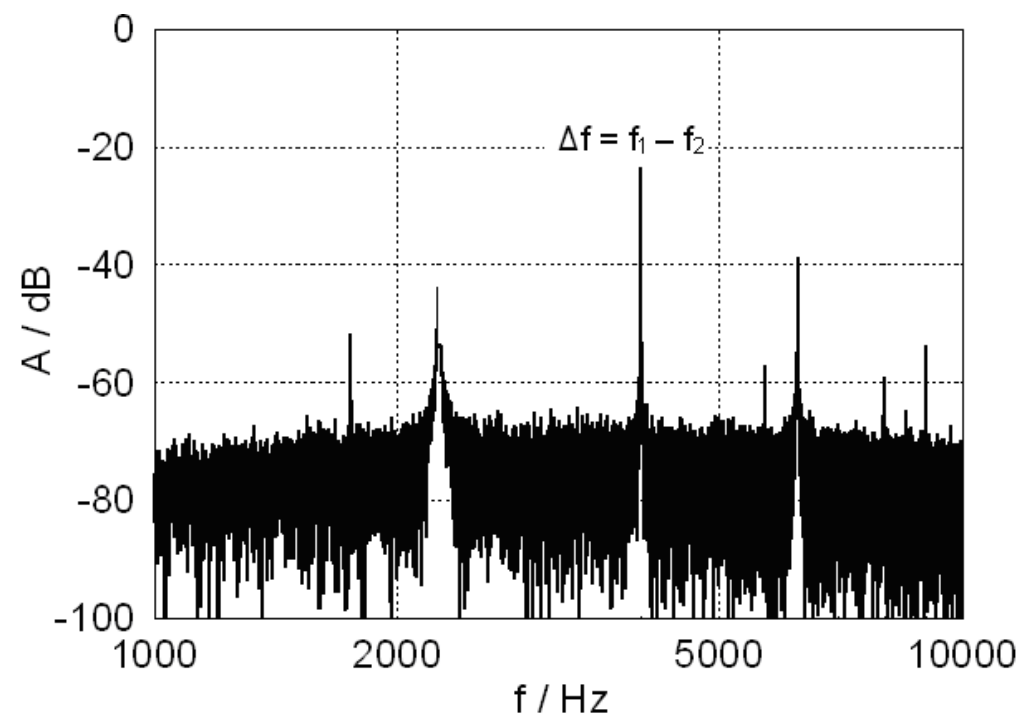

Figure 5: Frequency spectrum for No. 2 position and excitation signal frequencies 29 and $25 \mathrm{kHz}$. 
After the no-load girder measurement had been completed, the girder was loaded dynamically by means of steel plates of a mass of $320 \mathrm{~kg}$ each. By means of a UNC loader, three plates were made to fall down in succession from a height of $15 \mathrm{~cm}$ on a wooden pad, see Fig. 1 (on the right). The frame loaded the girder over the total of its length by means of two Ishaped joists, placed at a distance of $17 \mathrm{~cm}$ from each other on a NAIP cardboard of a thickness of $1.5 \mathrm{~cm}$. The response to the mechanical impulse was simultaneously recorded by two sensors placed on both measurement positions (see Fig. 2b).

Figure 6 shows the frequency spectra of the response to the mechanical impulses resulting from the impacts of two and three plates as recorded by the sensors at No. 1 and No. 2 measurement positions. In order to make the comparison more apposite, the amplitudes are not in $\mathrm{dB}$ units. The comparison of the frequency spectra of Fig. 6 a) No. 1 measurement position - gives evidence of a shift of the dominant frequency from $2.5 \mathrm{kHz}$ (two plate impact) to $3.5 \mathrm{kHz}$ in the case of the three plate impact. In the latter case, a much larger spread of the frequency spectrum values is seen when compared with the two-plate-impact.

Fig. 6 b) shows similar results for No. 2 measurement position. In this case again the high intensity of the exciting signal brought about an upward shift of the dominant frequency (from $2.5 \mathrm{kHz}$ to $4.7 \mathrm{kHz}$ ), the value spread of the frequency spectrum having become larger.

The loading process having been completed, the two harmonic exciting signals methods were applied. The results of the single harmonic ultrasonic signal excitation method application are shown in Fig. 7.
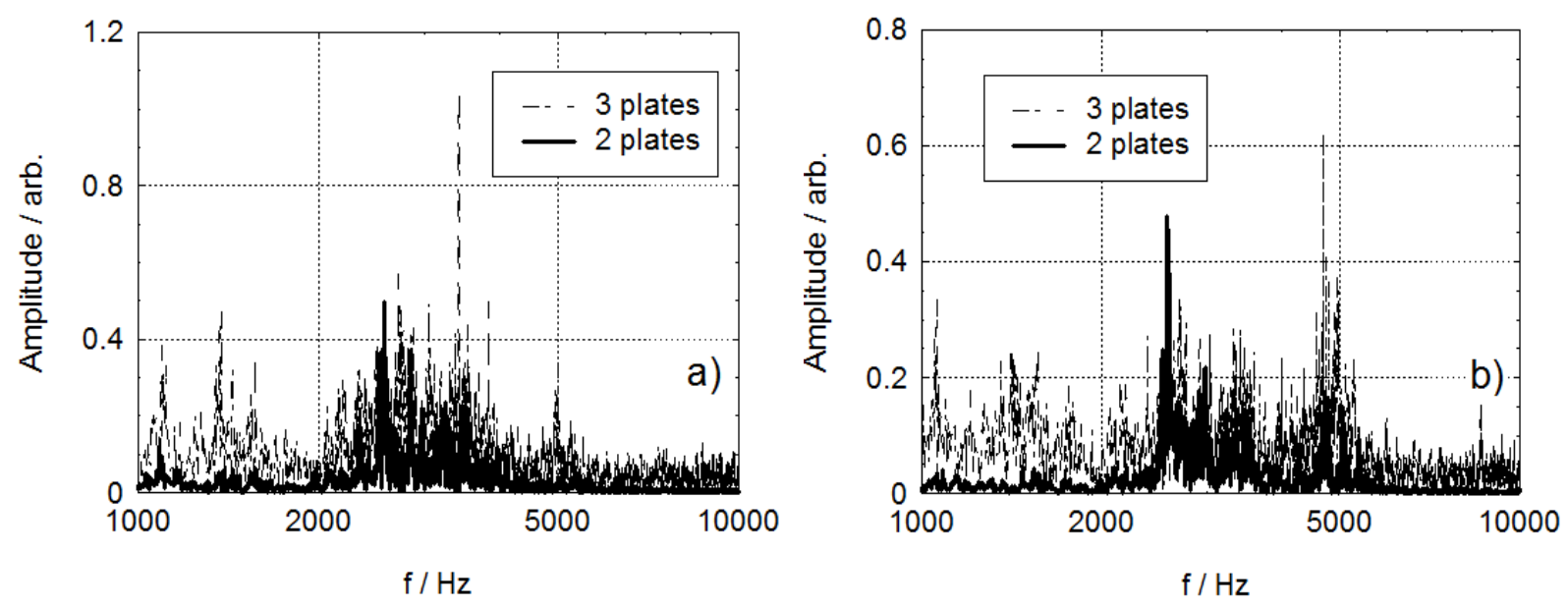

Figure 6: Frequency spectra of the response to the mechanical impulses:

a) No. 1 measurement position, b) No. 2 measurement position. 

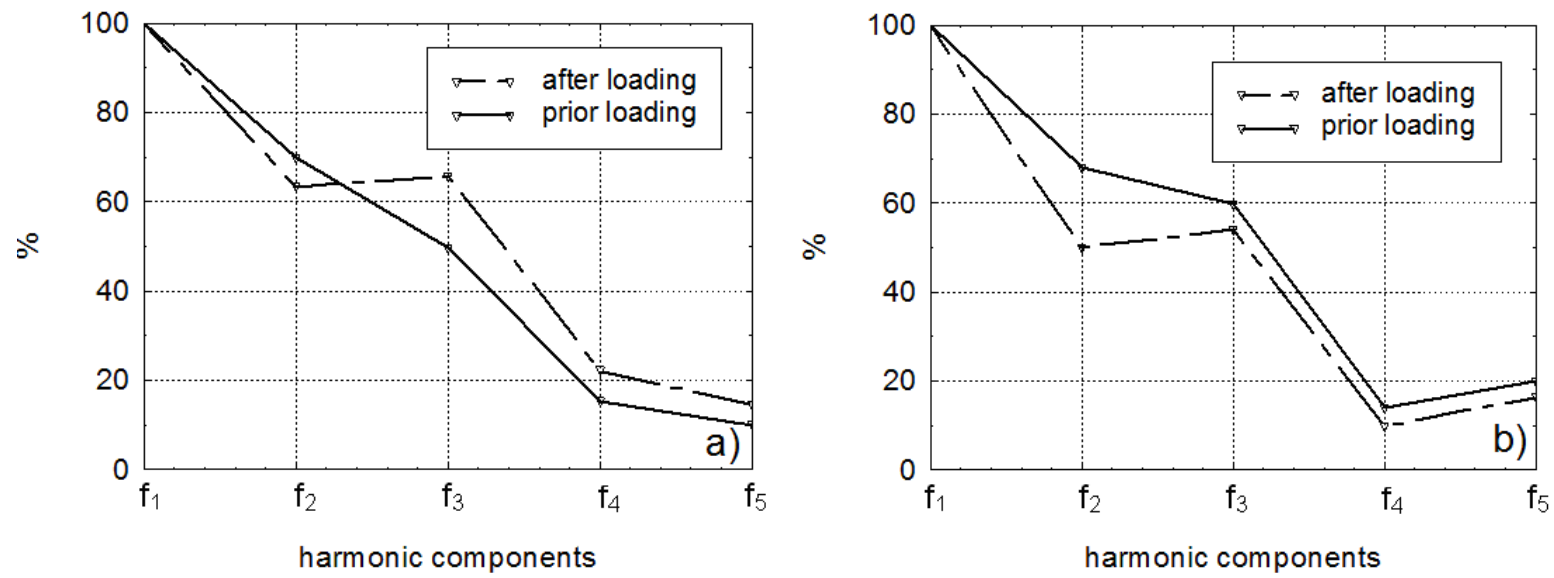

Figure 7: Higher harmonic frequency amplitudes expressed as a percentage of the first harmonic amplitude:

a) measurement position No. 1, b) position No. 2 .

Relative values of the higher harmonics obtained from No. 1 position prior to and after the girder loading are shown in Fig. 7 a). Nonlinear effects are evident to occur in the postloading curve. The amplitude of the third harmonic (frequency $f_{3}$ ) exceeds that of the second one (frequency $f_{2}$ ). Fig. 7 b) shows similar results for No. 2 measurement position. Both curves exhibit nonlinear effects. Similarly, the correlation coefficient squared shows a decrease in values compared to the pre-loading results, see Fig. 8.

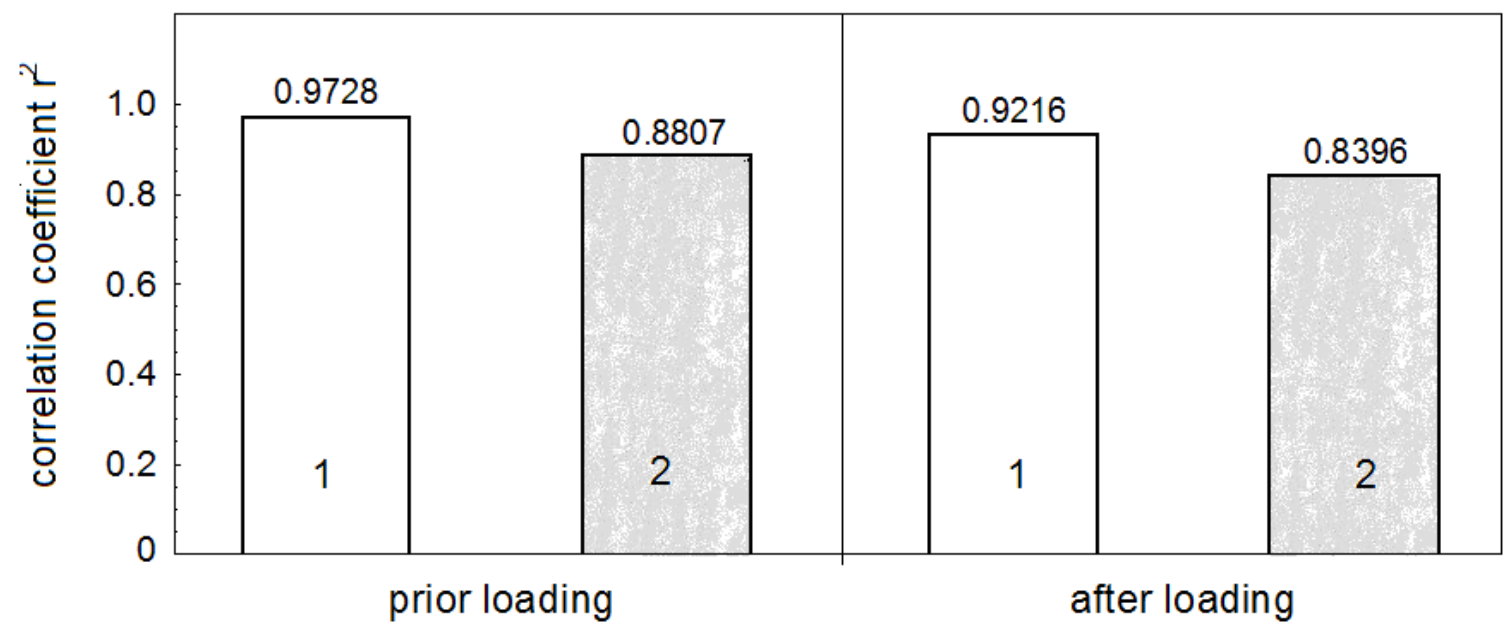

Figure 8: Variation of the correlation coefficient square for the first three harmonic frequency amplitudes.

Fig. 8 compares graphically the correlation coefficient squared values for both measurement positions $(1,2)$.

When applying the double harmonic exciting signal method, the measurement results are analogous. In both frequency spectra (measurement position No. 1 and 2), difference components $\Delta f=4 \mathrm{kHz}$ and their second harmonic component of a frequency of $8 \mathrm{kHz}$ can be identified, among more numerous parasitic components, see Fig. 9. 


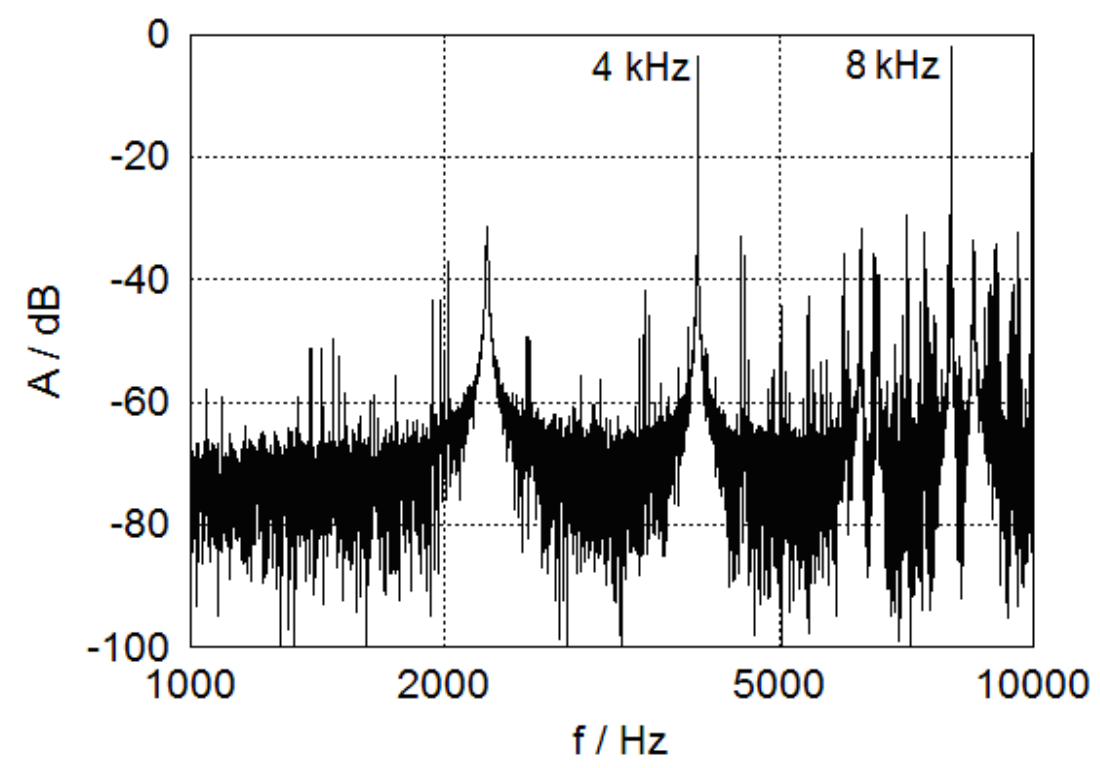

Figure 9: Frequency spectrum for No. 2 position and excitation signal frequencies 29 and $25 \mathrm{kHz}$, after loading.

\section{CONCLUSION}

The objective of these experiments was to verify the applicability of the nonlinear ultrasonic spectroscopy to monitoring the damage development in bulkier building elements in situ. A reinforced concrete KA girder, which had been extracted from the bridge structure in the course of the bridge reconstruction, was tested.

Measurement results are presented for two measurement positions which differ from each other in the steel armature pattern. Two nonlinear ultrasonic spectroscopy methods were applied to test the structure quality, namely, the single and the double harmonic ultrasonic signal methods. The measurement was carried out prior to the girder loading, the response was picked up during the girder dynamic loading and finally, the measurement was carried out after the girder loading was completed.

When analyzing the measurement results obtained from the single exciting harmonic ultrasonic signal method, we found that the damaged structure caused the amplitudes of oddnumbered harmonic components to increase in comparison with even harmonic components and the values of the square of the correlation coefficient to decrease. The occurrence of a new component whose frequency equalled the difference of the exciting frequencies in the frequency spectra obtained when using two exciting signals served as a nonlinearity and structure damage indicator. The above nonlinear effects were observed while measuring at the second measurement position prior to loading and at the both measurement positions after the load application. Steel plates falling down onto the girder were employed to implement dynamic loading of the girder. The girder response was picked up simultaneously at both measurement positions. The response frequency analysis as recorded for two different excitation intensities gave evidence of frequency changes at both measurement positions, which was due to the structure integrity defects.

The experiments were a continuation of the large laboratory measurements. The existing measuring set-up, which was assembled for laboratory testing, was supplemented and optimized; a high-frequency power amplifier was added for more extensive usage. The novelty of the experiments is the use of nonlinear ultrasonic spectroscopy methods to monitor structure integrity damage in bulkier structural elements in situ. Based on our 
analyses, we may state that the application of the nonlinear ultrasonic spectroscopy methods appears to be a promising approach to assessing the reinforced concrete structure integrity. Continual or repeated monitoring in specified time intervals and subsequent analysis of selected parameter variations will provide information on structure changes taking place in the building object under investigation.

\section{ACKNOWLEDGMENTS}

The research described in this paper is being solved within framework of the project No. P104/10/1430 by the Grant Agency of the Czech Republic.

\section{REFERENCES}

Aleshin, V., Van Den Abeele, K., 2007. Microcontact-based theory for acoustics in microdamaged materials. J. Mech. Phys. Solids, 55 (2), pp. 366-390.

Antonaci, P., Bruno, C. L. E., Gliozzi, A. S., Scalerandi, M., Bocca, P. G., 2010. Evolution of damage-induced nonlinearity in proximity of discontinuities in concrete. Int. J. Solids Struct., 47 (11-12), pp. 1603-1610.

Bentahar, M., El Aqra, H., El Guerjouma, R., Griffa, M., Scalerandi, M., 2006. Hysteretic elasticity in damaged concrete: Quantitative analysis of slow and fast dynamics. Phys. Rev. B, 73 (1), pp. 1-10.

Claytor, K. E., Koby, J. R., Ten Cate, J. A., 2009. Limitations of Preisach Theory: Elastic aftereffect, congruence, and end point memory. Geophys. Res. Lett., 36 (6), pp.1-4.

Hajek, K., Sikula, J., Sedlak, P., 2003. Experimental methods for nonlinear ultrasonic spectroscopy (analysis and application). In Proceedings of $33^{\text {rd }}$ International Conference Defektoskopie 2003, Ostrava (Czech Republic), November 19-21, 2003, pp. 65-72.

Haupert, S., Johnson, P. A., Laugier, P., 2011. High-accuracy acoustic detection of nonclassical component of material nonlinearity. J. Acoust. Soc. Am., 94 (5-6), pp. 277-302.

Korenska, M., Manychova, M., 2010. Study of New Posibilities of Non-destructive Testing of Ceramics Specimens Integrity. Ceramics-Silikaty, 54 (1), pp. 72-77.

Korenska, M., Matysik, M., Pospisil, K., 2008. NDT of Reinforcement Corrosion Using Ultrasonic Spectroscopy. Transactions on Transport Sciences, 1 (4), pp. 203-210.

Manychova, M., 2009. The Possibilities of Nonlinear Ultrasonic Spectroscopy for the NDT in Civil Engineering. Transactions on Transport Sciences, 2 (4), pp. 158-165.

Nazarov, V. E., Radostin, A. V., Ostrovsky, L. A., Soustova, I. A., 2003. Wave Processes in Media with Hysteretic Nonlinearity. Part I. Acoustical Physics, 49 (3), pp. 344-353. 
Shuia, G., Kim, J. Y., Qu, J., Wang, Y. S., Jacobs, L. J., 2008. A new technique for measuring the acoustic nonlinearity of materials using Rayleigh waves. NDT \& E Int., 41 (5), pp. 326-329.

Zumpano, G., Meo, M., 2008. Damage localization using transient nonlinear elastic wave spectroscopy on composite structures. Int. J. Nonlinear Mech., 43 (3), pp. 217-230.

The article was produced under the support of the project

Transport R\&D Centre (CZ.1.05/2.1.00/03.0064) 